\title{
EL EFECTO DIDÁCTICO EN CIENCIAS EXPERIMENTALES
}

\author{
Rómulo Gallego Badillo y Royman Pérez Miranda ${ }^{1}$ \\ Maestría en Docencia de la Química. Bogotá, Colombia \\ rgallego@uni.pedagogicaeduco royman@un.pedagogica.edu.co \\ Luz Nery Torres de Gallego y María Nelly Mantilla Mantilla \\ Colegio Jorbalán, Chía, Cundinamarca, Colombia.
}

\begin{abstract}
The partial results obtained in our research The interpretative, propositive and argumentative competencies in Science: A pedagogical and didactical problem" have suggested the need to apply the concept of didactic effect". The propositions here outlined fit into the non-lineal dynamics and into the theory of complexity.
\end{abstract}

Didactics; teaching of sciences; Didactic effect; linearity; Non-linearity; Compiexity.

\section{RESUMEN}

Del proyecto de investigación "Las competencias interpretar, argumentar y proponer en ciencias: Un problema pedagógico y didáctico", frente a los resultados parciales conseguidos, ha surgido la necesidad de acudir al concepto de "efecto didáctico". Los planteamientos que aquí se hacen al respecto, se enmarcan en la dinámica no lineal y en la teoría de comp iej i dad.

Didáctica; Enseñanza de las ciencias; Efecto didáctico; Linealidad; No Linealidad; Complejidad.

\section{INTRODUCCIÓN}

Los investigadores en la enseñanza de las ciencias experimentales, vienen sosteniendo la emergencia de la didáctica como una disciplina científica racional (Aliberas, Gutiérrez e Izquierdo, 1989). Aquellos que han optado por compromisos constructivistas, proponen una didáctica científica y metodológica, en la cual dejan de lado restricciones algorítmicas (Gil y colab. 1999), a la vez que delimitan el campo especifico de investigación en ella (Gil, Carrascosa y Martínez. 1999).

Para distinguir entre una didáctica metodológica y otra algorítmica, hay que sostener que esta última se enclava en una mirada exclusivamente instrumental y se reduce a la aplicación de métodos o pasos lineales y coherentes, para alcanzar unos objetivos; métodos o pasos que pueden ser seguidos por un tecnofacto (Gallego Badillo, 1995). La metodológica, es la que parte de una estructura conceptual (Gil y Carrascosa, 1985), de carácter hipotético-

\footnotetext{
${ }^{1}$ Profesores Universidad Pedagógica Nacional.
} 
deductivo, a partir de la cual se formulan hipótesis o se adelantan resultados, explicitándose las condiciones iniciales, el ordenamiento requerido, las intervenciones racionales los controles sobre las mismas a más de las regulaciones indispensables.

Aparece, entonces, el problema de la relación de proporcionalidad causa-efecto, y la diferenciación que es menester hacer entre sistemas lineales y no lineales, sobre todo si del cumplimiento o no de lo predicho se trata. SE acude, por consiguiente, a lo establecido por H. Poincaré (Hayles, 1990). Sostuvo él que el cumplimiento o no de una predicción, depende de si el sistema sigue o no esquemas repetitivos y si obedece o no a pautas discernibles. Puntualizó, además, que a mayoría de los sistemas dinámicos complejos no responden a la dinámica lineal.

Se estudian las relaciones de causalidad en el proceso de enseñanza-aprendizaje (Gallego Badillo, Pérez Miranda y Torres de Gallego. 1994). en razón de que se está frente a una didáctica científica y metodológica. hipotético - deductiva, por lo que los resultados del trabajo docente podrían asumirse en términos de contrastación positiva o negativa (Lakatos, 1983), de la estructura conceptual desde la cual se predijeron.

La producción o no del efecto didáctico, tal cual como ha sido caracterizado con antelación, ha de desprenderse de causas igualmente didácticas y obedecer o no a la equivalencia de las relaciones de proporcionalidad establecidas entre ambos; lo que a su vez, se halla ligado a la asimilación que se haga del colectivo aula y de las actividades que en él se propician, a la linealidad o no-linealidad..

En una investigación anterior (Pérez Miranda y Gallego Badillo, 2000). se demostró que los profesores encuestados parecían presentar imprecisiones en el concepto de aprendizaje o se referían a él, casi siempre, dentro del paradigma de la repetición al pie de la letra de lo enseñado; un aprender en el que la enseñanza tampoco era delimitada como su causa.

El cumplimiento exacto de la relación de proporcionalidad se daría, por ejemplo, en el caso de la repetición al pie de la letra de la información transmitida por el docente, en el cual la cantidad memorizada de ella debe ser equivalente a la enseñada; repetición que no debe ser cualquiera: ha de ser acertada, no equivocada, total, completa, y exacta, en cuanto se espera que coincido "punto a punto" con lo transmitido por el docente (Gallego Padilla, 1989).

Desde la propuesta del concepto de "efecto didáctico", la didáctica científica y metodológica, aludiendo a T. S. Kuhn (1972), se constituye en un cambio paradigmático, que no sólo instauro una nueva mirada, sino que también, inaugura reglas para la práctica del desempeño docente. Es acerca de esto que se discurre en el presente articulo.

\section{EL EFECTO DIDÁCTICO}

Es el conjunto de resultados específicos, productos de una enseñanza dada, demostrables mediante registros o datos obtenidos con instrumentos, validos y confiables: instrumentos formulados y aplicados para la contrastación empírica de la ocurrencia del efecto, tal como es anticipado, en términos de transformaciones cognoscitivas, afectivas y actuacionales por parte de os estudiantes (Gallego Badillo, 1999), o de los cambios esperados en sus estructuras conceptuales, metodológicas estéticas, actitudinales y axiológicas (Gallego 
Padilla y Pérez Miranda, 1998), o de las modificaciones en sus significados, formas de significar, de atribuir sentido y de actuar sistemática y congruentemente, de conformidad con las normas y reglas de la respectiva comunidad científica (Kuhn, 1972).

Referirse a los cambios en las estructuras conceptuales, metodológicas estéticas, actitudinales y axiológicas, es introducirse en la complejidad (Furia Más, 1996), y tomar distancia de la visión ingenua que se queda en el conceptualismo (Pozo, 1996); lo actitudinal, a su vez, reclama precisiones y distinciones necesarias (Furió Más y Vilches, 1997).

La complejidad, que no se queda en la sola enumeración de esas categorías, emerge de las interacciones entre ellas; de estas con el contenido curricular objeto de enseñanza y de estudio; con la información accesible al respecto; con las cognoscitivas que se desprenden de las controversias entre los miembros del colectivo aula; y, con otras que pueden surgir en la institución educativa y en el medio extra escolar ¿Qué peso proporcional de cada una de esas interacciones incide más en el cambio de las estructuras de la de cada uno de los integrantes del colectivo aula?

La demostración de que el electo didáctico se produjo y que tuvo como causa la enseñanza planeada, es un problema que se ha prefigurado como apoyo empírico (Lakatos, 1983) de la estructura conceptual y metodológica de partida. En este sentido, Se constituye en una contrastación experimental positiva o negativa y. en consecuencia, en fundamento de la comparación con otras teorías didácticas rivales (Popper, 1962). Incluso, esa estructura convertirse en un programa de investigación científica regresivo (Lakatos, 1983).

En la actualidad, por otro lado, se está generando una versión epistemológica nueva, que no contradice la constructivista, en la cual las teorías de las ciencias experimentales y de las tecnologías, son asumidas como sistemas de producción de mas y mejor saber especializado, a la vez que de mercancías; no sólo referidas a los tecnofactos, sino también a la información misma que se genero. Dentro de esta concepción, los profesionales de esas ciencias y de esas tecnologías, se están convirtiendo en trabajadores calificados del sistema.

Para los didactas constituye un reto ¿De qué manera las teorías de su disciplina científica y metodológica podrían ser asumidas como sistemas de producción de más y mejor saber didáctico? ¿Qué se requeriría para que la información especializada y procedente de la investigación, se convirtiera en mercancía? ¿Cuáles serian los tecnofactos que se desprenderían de esa investigación? ¿Cómo hacer de los docentes clientes potenciales? ¿Cuáles serían las características del mercado de esos productos? Quiérase o no, en el mundo actual, ello significaría contar con apoyo financiero (Di Trocchio, 1995), para la continuidad de la comunidad de investigadores en la enseñanza de las ciencias experimentales.

A partir de esta visión, se impone la exigencia de formular proyectos curriculares Centrados en la construcción de competencias cognoscitivas (Gallego Badillo, 2000). En el caso de Colombia, el nuevo examen de estado obligatorio para todos los egresados de la Educación Media Vocacional, ausculta en sus pruebas las competencias interpretar, argumentar y proponer. El problema que surge es aquel en el que toda interpretación, para serlo, tiene que ser necesariamente nueva, ya que de no ser así, se tendría una repetición de lo afirmado por otros; en la particularidad de las ciencias experimentales, la argumentación, para su sostenibilidad, ha de apoyarse, en hechos concretos de laboratorio o en 
experimentos mentales; la construcción de la competencia propositiva, se expresaría en la elaboración de consecuencias teóricas y prácticas de la interpretación y la argumentación hechas.

Una enseñanza en ciencias centrada en la construcción de competencias cognoscitivas, parece ser la exigencia de las políticas educativas vigentes, hasta el punto de que se están proponiendo pruebas internacionales basadas en dicha construcción, con miras a establecer comparaciones y emitir juicios relativos a la calidad y a la excelencia de esa enseñanza. Esto constituye un desafío para los practicantes de la didáctica científica y metodológica. Los contenidos curriculares han de ser reestructurados entonces, para que, además de transformar las cosmovisiones, sirvan para la producción.

Interpretar, argumentar y proponer, remiten al viejo problema didáctico de enseñar a leer y a escribir en el contexto conceptual y metodológico de la disciplina objeto de enseñanza. Un leer que no se queda en la decodificación, puesto que ha de extenderse a interpretar información especializada; Un escribir, en razón de que la tradición científica y académica, no es oral.

Si toda interpretación es una elaboración original de cada uno de los miembros del colectivo aula es, en consecuencia, producto de su creatividad, la cual trabaja a partir de las estructuras de significados, de formas de significar, de atribuir sentido y de actuar preexistentes. Para que esa creatividad emerja la actividad cognoscitiva tiene que ser desplegada a través de una dinámica no-lineal. En esta situación, el efecto didáctico adelantado puede no producirse o ser sorprendente; efecto este desde el cual interpretar, argumentar y proponer se lleva a cabo a partir de una cognición con bases diferentes

\section{EL PROBLEMA DE LA CONTRASTACIÓN}

En principio y siguiendo convenciones de la comunidad científica, tan criticables como se quiera, dentro de las aproximaciones constructivistas que orientan estas puntualizaciones, se postulo que la contrastación a la que se alude, sólo es factible si los instrumentos de registro o de recolección de información, se formulan y se aplican, parlo menos, antes y después de realizado el trabajo didáctico alrededor de una unidad temática particular, ya que es así como se pueden hacer comparaciones sustentadas y emitir un juicio consecuente acerca de si el efecto didáctico planeado y esperado se produjo y fue causado por las estrategias de enseñanza puestas en práctica.

Se trata de una planeación que delimité las condiciones de partida, las caracterizó en lo conceptual, metodológico, estético, actitudinal y axiológico y que, hipotéticamente especificó las finales en los mismos términos; además estableció el ordenamiento requerido del ámbito didáctico, las intervenciones metódicas y las regulaciones racionales y concertadas con los estudiantes, que se pusieron en práctica. Así, la contrastación parte de unos registros o informaciones iniciales y termina con unos finales, para hacer las comparaciones y considerar si el efecto se produjo o no.

Subráyese en lo afirmado, que tomar partida parlo metódica de la aplicación inicial y final de las pruebas, tiene compromisos señalables, los cuales tratarían al colectivo aula y a quienes lo conforman, como un sistema que parte de un estado de salida, para desplazarse 
a otro de llegada, por lo que se renunciaría a considerar ese movimiento como un proceso y la didáctica científica y metodológica no tendría la necesidad de explicar las evoluciones cognoscitivas seguidas, lo que dejaría sin fundamentación teórica, las intervenciones, los controles y las regulaciones indispensables para la obtención del efecto. Se podría regresar a la versión instrumental o artesanal (Koyré, 1979), en la que para la práctica docente, basta con un entrenamiento.

Siguiendo con lo postulado habría que sentar de antemano si la organización del ámbito didáctico y las actividades cognoscitivas que en su seno se impulsan, apoyan y canalizan seguir una dinámica lineal o una no-lineal; determinación ésta que incidiría en la clase de estrategias de enseñanza, de conformidad con el efecto didáctico que se busca. En el primer caso, treme a un estado cognoscitiva inicial (Eí), el efecto seria un estado final (Ef), como un esquema repetitivo que siguiera pautas discernibles, sin que se rompiera la simetría temporal (Prigogine y Stenger, 1990), puesto que no habría elaboración de nuevos conocimientos, expresables estos en la construcción de las competencias interpretar, argumentar y proponer.

La no ruptura de la simetría temporal significaría que pese al trabajo didáctico realizado, los estudiantes cognoscitiva, afectiva y actuacionalmente continúan siendo lo que antes fueron. Siguen siendo.

En el segundo, se abrirían muchas posibilidades, tanto al colectivo aula, coma a cada uno de sus integrantes, hasta el punto de que se puede hablar de la probabilidad de que se den, discriminadamente, los estados, Ef1, Ef2, Ef3..., Efn; existiendo el problema, dada la creatividad inherente a la actividad cognoscitiva, de que no se pueda especificar de antemano, cuál de esos estados se construirá coma tal, o que seleccionado uno de ellos como el efecto esperado, no ocurra, obteniéndose otra sorprendente. No obstante, se habrá producido una transformación en las estructuras conceptuales, metodológicas, estéticas, actitudinales y axiológicas de los estudiantes, por lo que serán distintos, cognoscitiva, afectiva y actuacionalmente rompiéndose la simetría temporal.

Si el efecto didáctico se produce a través de una dinámica no lineal, es sorprendente y no siguió la relación simple de proporcionalidad causa-efecto ¿De qué manera ese suceso no esperada apoya o no la estructura conceptual y metodológica de la didáctica científica desde la que se delimitaron las condiciones iniciales, se ordenó el ámbito de enseñanza y se decidieron las intervenciones, los controles y las regulaciones?

Puesto que el análisis de la comparación entre los registros o los datos suministrados parlas pruebas iniciales y finales, persigue contrastar la estructura conceptual y metodológica de la didáctica metodológica que fundamenté la enseñanza, como un trabajo de investigación; tal análisis ha de guiarse pon interrogantes como; ¿Se produjo un cambio en lo cognoscitiva, lo metodológico y lo actuacional? ¿Ese cambio fue el esperada? ¿En qué medida se produjo? ¿Fue consecuencia de las estrategias aplicadas? ¿Incidieran factores no considerados? ¿De qué manera el efecto didáctico apoya o no empíricamente la estructura conceptual y metodológica de partida que fundamenté el proceso? ¿Qué consecuencias teóricas y prácticas acarreo?

Digase, de paso, que esta didáctica establece e inaugura la exigencia de que, en el campo de la enseñanza de las ciencias experimentales, los profesionales de esa praxis, no 
continúen basando sus explicaciones y dando cuenta de sus desempeños, mediante apreciaciones no sostenibles y, por tanta, no ubicadas en la problemática de la relación de proporcionalidad entre las causas didácticas que hipotéticamente establecen y los efectos didácticos que han de darse necesariamente.

\section{DE LAS ESTRATEGIAS DE ENSEÑANZA}

En el interior de esa didáctica científica y metodológica. que rompe con miradas instrumentales y artesanales, el problema de formular y llevar a la práctica unas estrategias de enseñanza, se configuro como parte integrante de su campo específico de investigación si de la no linealidad se trata.

Dicho problema se concreto en el cómo de a intervención y del control que se precisa hacer sobre las estructuras iniciales de los estudiantes para que, a partir de ese control y de esa regulación, se impulse se apoye y se propicie la actividad cognoscitiva con miras a la producción de un efecto didáctico pre-establecido, que puede cumplir-se o no. Desde este punto de vista no existirán estrategias didácticas establecidas de antemano como fórmulas genéricas.

\section{LOS FUNDAMENTOS DE LAS PRUEBAS}

Dentro de la educación institucional, regida por un proyecto curricular con unas intencionalidades que delimitan la razón de la enseñanza de las ciencias experimentales en cada nivel del sistema educativo del que se trate, el efecto didáctico puede ser inicialmente establecido, de manera general. A partir de esta primera puntualización sigue un proceso de consulta y de reflexión que ha de desembocar en unas precisiones, exigibles por esa didáctica científica y metodológica.

Para este cometido, es posible acudir, hasta cierto punto, a la geometría fractal (Mandelbrot, 1983), por lo que lo cognoscitivo seria afectivo y actuacional, lo afectivo cognoscitivo y actuacional, a la vez que lo actuacional gozaría también de ser cognoscitivo y afectivo, es decir, cada uno se realiza a través de las dimensiones de los otros. En este orden de ideas, habría que pensar en que cada uno de ellos es desplegable en subdimensiones, como serian las conceptuales, las metodológicas, las estéticas, las actitudinales y las axiológicas, por lo que las pruebas sobre lo cognoscitivo, lo afectivo y lo actuacional, habrían de formularse en sus items, para auscultar cada uno de los componentes de tales subdimensiones.

La especificación de tales componentes, ha de apoyarse en lo establecido por la intencionalidad curricular, tanto como en los contenidas de la unidad objeto de trabajo didáctico y, por supuesto, en las concepciones epistemológicas que se tienen en torno a la disciplina científica a la que pertenece dicha unidad.

Basarse para ello, en primer lugar. en las revistas de la comunidad científica correspondiente y, en segundo lugar, en los textos de enseñanza disponibles en el mercado bibliográfico; algo que hace aparecer el problema de la confiabilidad en esos textos. 
Precisado el efecto didáctico y delimitada la estructura conceptual y metodológica de la unidad objeto de enseñanza y de estudio por parte del colectivo aula, ha de procederse a la construcción de la enseñabilidad (Gallego Badillo y Pérez Miranda, 1999) de la misma, lo que se plasma en la estructuración didáctica necesaria y correspondiente, de la cual se han de derivar las estrategias de enseñanza y, por supuesto, la formulación específica de las pruebas iniciales y finales esa estructuración. En esta perspectiva, las pruebas no existen antes, en una hipotética batería de instrumentos aplicables en cualquier caso, independientemente de la transformación cognoscitiva a la que se apunta, cualesquiera estudiantes y unidades didácticas sean. Los instrumentos de registro o de recolección de información son así didácticos, porque la estructuración interna que se les atribuye obedece a los conceptos y metodologías propias de esta disciplina científica, de acuerdo con los propósitos de la contrastación, las características de lo cognoscitivo, lo afectivo y de lo actuacional objeto de esa transformación.

Finalmente, y dada la complejidad del objeto de estudio y enseñanza y a la no linealidad de la dinámica con la que se le hace ¿parecer ese objeto, es claro que no basta con la formulación y aplicación de un sólo y único instrumento, dado que han de ser por lo menos tres, cuando no cinco, dependiendo de las transformaciones cognoscitivas, afectivas y actuacianales, en sus subdimensiones conceptuales, metodológicas, estéticas, actitudinales y axiológica. Si se considera lo cotidiano de las exigencias del desempeño docente, tres pruebas podrían ser necesarias y suficientes.

Contrariamente a las concepciones y prácticas habituales en torno a la enseñanza de las ciencias, en las que la evaluación se constituye en un evento al final $d$, e independiente y exógeno al proceso didáctico adelantada por los docentes, en el que la relación de causalidad no se considera, la formulación y aplicación de pruebas antes y después constituye en sí parte fundamental de las estrategias de enseñanza, ya que, si a las iniciales se atiende, la construcción de sus items ha de ser en sí didáctica, para convocarlos e introducirlos en la reflexión sistemática y coherente sobre de lo que ya saben al respecto, e iniciarlos en la dinámica no lineal de la transformación de eso que ya saben y desde lo cual interpretan, argumentan y proponen.

\section{DE LOS REGISTROS O DATOS}

El interrogante se centra en preguntar por las exigencias de que estos sean sólo cualitativos o cuantitativos. Al respecto, J. W. Ratcliffe (1983), precisa que la última característica tiene como basamento una delimitación primera e indispensable, de tipo cualitativo, en la medida en que ha de partir de delimitaciones acerca de aquello a lo que se hace referencia, en este caso de la ocurrencia o no del efecto didáctico.

Por otro lado, si se atiende a la lógica de la estructura de los conceptos científicos (Mosterin, 1978), cabria decidir si la didáctica científica y metodológica trabaja a no con conceptos cualitativos o clasificatorios, o con comparativos o con métricos o cuantitativos, vale decir, con magnitudes; caso este último en el que se podría hablar, con propiedad, de medida o de procesos de medición. Ello supondría una geometrización, matematización y metrización de aquello que seria objeto de medición. 
Sigue lo estadístico, que apareja la delimitación estricta de un espacio muestral, en el cual, por los postulados de tal disciplina, cada punto del mismo tiene que ser necesariamente independiente de los otros ¿Qué idealización extrema hay que hacer? Si cada miembro del colectivo aula interactüa cognoscitivamente con los demás integrantes del mismo ¿Desde qué didáctica se justificaría la aplicación estadística? ¿Impedir esas interacciones? Atendiendo a la convicción de que las ciencias son construcciones comunitarias (Hodson, 1985) ¿Qué indagan, qué explicitan y a qué dan pie los datos estadísticos para elaborar imerpretaciones didácticas?

Frente a los cuestionamientos establecidas, se podría acudir al principio de que, desde la perspectiva matemática, el procedimiento básico es el de contar. Referido ello a la estructura conceptual y metodológica de la unidad objeto de enseñanza y de estudio, cada prueba habría de precisar, con base en la estructuración didáctica, los conceptos fundamentales, delimitar los básicos y los derivados, a la vez que establecer las respectivas jerarquías lógicas entre ellos, como la admisibilidad científica de las mismas, en lo que radicaría, además, el problema de la formulación, aplicación e interpretación de los registros o datos obtenidas con las pruebas.

\section{CONCLUSIONES}

Como puede notarse, la propuesta del efecto didáctico, dentro de las consideraciones lineales o no lineales, se configuro como un argumento que apoya la constitución de la didáctica científica y metodológica. A su vez, introduce en la reflexión acerca del problema de la formulación de las estrategias de enseñanza, como también de la elaboración de las pruebas que han de suministrar los registros o los datos para efecto de las contrastaciones exigibles e indicativa de la afectividad de dicha didáctica.

Prefigura un marco referencial de evaluación para proyectos de investigación, tesis o trabajos de grado, en orden a las exigencias de rigurosidad y coherencia indispensables. En cualquier caso abre una discusión antes no propuesta pero indispensable.

\section{REFERENCIAS}

Aliberas, J.; Gutiérrez, R. e Izquierdo, M. 1989. La didáctica de las ciencias: Una empresa racional. Enseñanza de las Ciencias, 7(3), 227-280.

Di Trocchio, F. 1 995. Las mentiras de la ciencia ¿Por qué y cómo engañan las científicos? Madrid: Alianza.

Punió Más, C. 1996. Las concepciones alternativas del alumnado. Dos décadas de investigación. Resultados y tendencias. Alambique. Didáctica de las Ciencias Experimentales, No. 7,7-17.

Furió Más, C. y Vilches, A. 1997. La dimensión afectiva del aprendizaje de las ciencias: Actitudes hacia las ciencias y relaciones ciencia, tecnología y sociedad". En: La 
enseñanza-aprendizaje de las ciencias de la naturaleza en la Educación Secundaria. Barcelona: I.C.E., Universidad Autónoma de Barcelona.

Gallego Badillo, R. 1999. Competencias cognoscitivas. Un punto de vista epistemológico, pedagógico y didáctico. Bogotá: Cooperativa Editorial Magisterio. Magisterio.

1995. Discurso constructivísta sobre las tecnologías. Bogotá: Cooperativa Editorial 1992. Comunidad de educadores. Construcción y dinamización. Santa Fe de Bogotá: Antropos.

1989. Evaluación pedagógica y promoción académica. Bogotá: ECOE.

Gallega Badillo, R. y Pérez Miranda, H. 1998. "Aprendibilidad-enseñabilidad-educabilidad: una discusión”. Revista Colombiana de Educación, Nos. 36 -37; 69-92.

.1999. Aprendibilidad, enseñabilidad y educabilidad en las ciencias experimentales". Educación y Pedagogía, No. 25,89-117.

Gallego Badillo, R.; Pérez Miranda, R. Y Torres de Gallego, L. N. 1994. "El problema de la causalidad en la relación enseñanza-aprendizaje”. Actualidad Educativa, Año 1, No. 1, 43-47.

Gil Pérez, D., Carrascosa Alís, J. y Martínez Terrades, F. 1999. El surgimiento de la didáctica de las ciencias como campo especifico de conocimientos". Educación y Pedagogía, No 25, $15-65$.

Gil Pérez y Colab. 1999. "¿Puede hablarse de consenso constructivista en la educación científica?”. Enseñanza de las Ciencias, 17(3), 503- 512.

Gil Pérez, D. y Carrascosa Allis, J. 1985. Science learning as conceptual and methodological change. Europ. Journ. Science Education, 7(3), 231-253.

Hayles, N. K. 1993. La evolución del caos. Barcelona: GEDISA.

Hodson, D. 1985. Phylosophy of science and science education. Studies in Science Education, 12(1), 25-27.

Koyré, A. 1979. Del mundo del "más o menos" al universa de la precisión. Naturaleza, Educación y Ciencia, No. 4,1 Semestre. Bogotá: Universidad Nacional de Colombia.

Kuhn, T. S. 1972. La estructura de las revoluciones científicas. México: Fondo de Cultura Económica.

Lakatos, I. 1983. La metodología de los programas de investigación científica. Madrid: Alianza. 
Mandelbrot, B. 1983. The fractal geometry of nature. New York, W. H. Frreman and Company.

Mostenín, J. 1978. "La estructura de los conceptos científicos". Investigación y Ciencia, No. $16,80-93$.

Pérez Miranda, R. y Gallego Badillo, R. 2000. Pedagogía y profesionalidad. Las oncepciones actuales. Memoria de investigación. Bogotá: Universidad Pedagógica Nacional, Centro de Investigaciones (CIUP).

Prigogine, I. y Stenger, I. 1990. La nueva Alianza. Metamorfosis de la ciencia. Madrid: Alianza.

Pozo, J. I. 1996. Las ideas del alumno sobre ciencia: De dónde vienen, adónde van y... mientras tanto qué hacemos con ellas". Alambique. Didáctica de las Ciencias Experimentales, No. 7,18-26.

Popper, K. 1962. La lógica de la investigación científica. Madrid: Editorial Tecnos.

Ratcliffe, W.J. 1983. Notíons of validity a qualitative research methodology. Knowledge, Creation Difution Utilization, vol. 5, No. 2,147-167. 